\title{
Motor Control-Learning Model for Reaching Movements
}

\author{
Hiroyuki Kambara, Kyoungsik Kim, Duk Shin, Makoto Sato, and Yasuharu Koike
}

\begin{abstract}
One of the great abilities of the central nervous system (CNS) is that it can learn by itself how to control our body to execute required tasks. Although several motor control models have been proposed to explain well-learned arm reaching movements, those models do not fully consider how the CNS learns to control our body. In this paper, we propose a new motor control model that can learn to generate accurate reaching movements without prior knowledge of arm dynamics. In our model, the control law is learned in a trial-and-error manner using the reward signal. We focus on point-to-point arm reaching task in the sagittal plane and show that accurate reaching movements toward any given point can be learned and generated by our model. Furthermore, the model can predict human subjects' hand trajectories without specifying desired trajectories.
\end{abstract}

\section{INTRODUCTION}

When we move our hand from one point to another, our hand paths tend to gently curve and the hand speed profiles are bell-shaped [1][2][3]. Since humans show those highly stereotyped trajectories among an infinite number of possible ones, it has been suggested that the CNS is optimizing arm movements so as to minimize some kind of cost function [3][4][5]. The cost function specifies what kind of variables should be minimized during the movement or after the movement. Several optimal control models have been proposed [6][7][8] to explain the way the CNS generates a set of motor commands that could minimize cost functions. The hand trajectories predicted by those models are in good agreement with experimental data. The purpose of these models, however, is to predict well-learned reaching movements and not to describe the process of learning.

The purpose of this paper is to propose a motor control model that could learn the control law (or control policy) for reaching movements while controlling the arm. Let us call this type of model "motor control-learning model". From observing infants' inability to generate adult-like smooth movement [9][10], it is clear that the CNS is somehow learning to control our body after we are born. However, this is not as easy as studying for an exam, since there is no explicit "teacher" that could provide the CNS with correct motor commands. Thus the CNS has to learn how to control the body in a trial-and-error manner, through interaction with the environment.

Reinforcement learning has attracted a lot of attention as a candidate mechanism for acquiring optimal control strategy through trial and error [11]. The Actor-Critic method

All authors are with Tokyo Institute of Technology, Yokohama, 2268503, Japan (phone:+81-45-924-5054; fax:+81-45-924-5016; email: hkambara@hi.pi.titech.ac.jp)

Hiroyuki Kambara, Duk Shin, Kyoungsik Kim and Yasuharu Koike are also with Japan Science and Technology Agency CREST

Hiroyuki Kambara is also with University of California San Diego in particular is one of the major frameworks for temporal difference (TD) learning, and has been proposed as a model of learning in the basal ganglia [12][13].

We adopt the Actor-Critic method [14] in order to acquire a feedback controller for arm reaching. Although we are not the first to apply the Actor-Critic method to reaching tasks, previous models only explain the reaching movement toward one particular target [15]. In our daily life, we are not always reaching to the same target. The CNS has to learn how to generate reaching movements towards any target in the workspace. However, it is hard to generate those various movements with high accuracy by a single feedback controller. Since the gravitational force acting to the arm is dependent on the arm posture, the force required to hold the hand at the target varies with the target position. Furthermore, muscle force varies with arm posture even if the same level activation is sent to the muscles. For these reasons, there is no guarantee that a feedback controller trained for a particular target would generate accurate reaching movements to other targets.

Here we introduce an additional controller called Inverse Statics Model (ISM), in order to help the feedback controller to generate reaching movements toward various targets. The ISM handles the static component of the inverse dynamics. That is, it transforms a desired equilibrium position (or posture) into a set of motor commands that could move the hand to the target and hold it there. Note that the arm converges to certain equilibrium posture when constant motor commands are sent to the muscles because of the spring-like properties of the musculoskeletal system [16]. If the ISM is learned properly, it could compensate the static forces (e.g. gravity) at the target point. Therefore, accurate reaching movements to various points are realized by combining ISM with a feedback controller acquired using the Actor-Critic method.

To learn the ISM in a trial-and-error manner, we adopted the Feedback-Error-Learning scheme [17]. In this scheme, the inverse dynamics (or statics) model of the controlled object is trained by using the feedback controller's output as an error signal. This learning scheme was originally proposed as a computational coherent model of cerebellar motor learning [17][18]. The original model, however, did not explain how to train the feedback controller. In our control-learning model, an Actor-Critic method is introduced to learn the feedback controller needed for the FeedbackError-Learning scheme [19]. To train an inverse dynamics model using Feedback-Error-Learning, a desired trajectory must be specified. The original model, however, did not explain how to calculate the desired trajectory. There is a neural network model for trajectory formation [20], but 
it requires a pre-trained inverse dynamics model. Thus, the trajectory formation problem in Feedback-Error-Learning remains to be solved.

An important advantage of combining reinforcement learning and ISM is that a desired trajectory is no longer needed. Note that the term 'trajectory' includes not only a sequence of positions but also sequences of velocities and accelerations. The musculoskeletal system is extremely complex, including non-linear dynamics and many redundancies. Therefore, complex calculation is often necessary to acquire the desired trajectory minimizing some cost function. In our motor control-learning model, the feedback controller is trained to maximize expected cumulative "reward" (which is the same as minimizing cost), and it drives the arm directly toward the target. The concept of using a goal-directed control strategy, instead of following a desired trajectory, has succeeded in predicting the features of several human movements [8]. On the other hand, the ISM also serves as goal-directed feedforward controller in our model. It receives the target position as an input signal and outputs a set of motor commands which shift the arm's equilibrium to the target. ISM in our model resembles the "Endpoint Control Hypothesis" which was proposed earlier [16][21]. This hypothesis was quite attractive for its simplicity. Unfortunately, it was rejected by later experiments [22] indicating that a desired trajectory is planned by the CNS. However, we show that the same experimental results can be qualitatively reproduced without assuming a desired trajectory, if we assume that a Forward Dynamics Model (FDM) of the arm is utilized in addition to the feedback controller and the ISM [23].

Although there is an argument whether the CNS is planning desired trajectory for well-trained tasks or not [8][24], our standpoint is that the CNS is not planning desired trajectories for our daily movements. In a previous paper we showed that our model could predict the hand paths of human point-to-point reaching movements in the sagittal plane without planning desired trajectories [19]. However, we could not reproduce the smooth bell-shaped hand speed profiles even though the ISM and feedback controller networks were parametrised with smooth Gaussian basis functions. The speed profiles showed steeper initial rise compared to data. The reason for these steep profiles seemed to be the deficit of smoothness in the muscle model. In neurophysiological studies, it is known that muscle forces could be well predicted by low-pass-filtering the neural impulses with a second-order filter [25]. In this paper we incorporate such a filter in the plant dynamics [26]. In addition, we introduce FDM in our motor control-learning model. FDM is the internal model that predicts the movement trajectory given outgoing motor commands [18][27]. In our model, FDM predicts future states and thus compensates the delays caused by the low-pass muscle filter.

In this paper, we apply our motor control-learning model to arm point-to-point reaching task in sagittal plane. We show that our model could reconstruct accurate reaching movements to various points in a trial-and-error manner. In

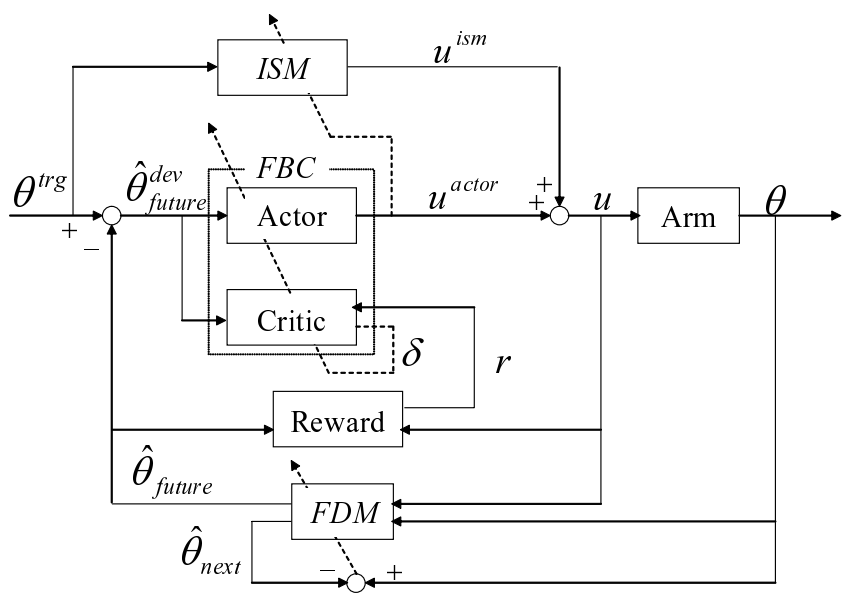

Fig. 1. The architecture of motor control-learning model: The model consists of Inverse Statics Model (ISM), Feedback Controller (FBC) and Forward Dynamics Model (FDM). FBC is composed of Actor and Critic, which are controller and state value function estimator respectively in ActorCritic method. ISM generates feed-forward motor command $\boldsymbol{u}^{i s m}$ whose equilibrium point becomes the target state $\boldsymbol{\theta}^{\text {trg }}$. On the other hand, Actor generates feedback motor command $\boldsymbol{u}^{\text {actor }}$ that can reduce the deviation between the target state $\boldsymbol{\theta}^{\operatorname{trg}}$ and the future state $\hat{\boldsymbol{\theta}}_{\text {future }}$ predicted by FDM. Actor's motor command $\boldsymbol{u}^{\text {actor }}$ is used as an error signal for the update of ISM. The reward signal $r$ is determined from the predicted future state $\hat{\boldsymbol{\theta}}_{\text {future }}$ and total motor command $\boldsymbol{u}$ which is the sum of $\boldsymbol{u}^{i s m}$ and $\boldsymbol{u}^{\text {actor }}$. Actor is updated so that it can generate motor command that maximize the value of cumulative reward. Critic is updated so that it can predict the value of cumulative reward. An error signal for the update of FDM is the deviation between predicted next state $\hat{\boldsymbol{\theta}}_{\text {next }}$ and the state $\boldsymbol{\theta}$ observed at the next time step.

addition we compare the model predictions to experimental data, and show that our model can predict the features of both hand path and speed profile of human reaching movements without planning desired trajectories.

\section{Motor Control-Learning Model}

Figure 1 illustrates the architecture of our motor controllearning model for arm's reaching task. The model has three main units, Inverse Statics Model (ISM), Feedback Controller (FBC) and Forward Dynamics Model (FDM). FBC is composed of Actor and Critic, which are controller and state value function estimator respectively in Actor-Critic method.

During each trial, target state $\boldsymbol{\theta}^{\text {trg }}$ is kept constant at the value of the target, and its velocity components are always 0 . ISM receives target state $\boldsymbol{\theta}^{\text {trg }}$ as input and outputs timeinvariant motor command $\boldsymbol{u}^{i s m}$. On the other hand, at every moment, Actor receives the deviation $\hat{\boldsymbol{\theta}}_{\text {future }}^{\text {dev }}$ between target state $\boldsymbol{\theta}^{\text {trg }}$ and future state $\hat{\boldsymbol{\theta}}_{\text {future }}$ predicted by FDM at one step time before. Actor, then, generates motor command $\boldsymbol{u}^{\text {actor }}$. The total motor command $\boldsymbol{u}$ is the sum of $\boldsymbol{u}^{i s m}$ and $\boldsymbol{u}^{\text {actor }}$. From the total motor command $\boldsymbol{u}$ and state $\boldsymbol{\theta}$, FDM predicts next state $\hat{\boldsymbol{\theta}}_{\text {next }}$ and also future state $\hat{\boldsymbol{\theta}}_{\text {future }}$.

The state of the $\operatorname{arm} \boldsymbol{\theta}$ is changed by the motor command $\boldsymbol{u}$, and the new state is used as a teaching signal to FDM. On the other hand, reward $r$ is determined by predicted future state $\hat{\boldsymbol{\theta}}_{\text {future }}$ and motor command $\boldsymbol{u}$. The temporal 
difference (TD) error $\delta$ is then calculated from reward $r$ and estimated state value of $\hat{\boldsymbol{\theta}}_{\text {future }}^{\text {dev }}$. Critic and Actor are updated by using TD error $\delta$ as an error signal and reinforcer respectively. Finally, Actor's motor command $\boldsymbol{u}^{\text {actor }}$ is used as an error signal for ISM learning.

Now let us describe the detail of each three units.

\section{A. Actor-Critic}

The inputs to Actor and Critic are both $\hat{\boldsymbol{\theta}}_{\text {future }}^{\text {dev }}(t)$. Let us denote this $\hat{\boldsymbol{\theta}}_{\text {future }}^{\text {dev }}(t)$ simply as $\boldsymbol{x}(t)$ for compact notation $\left(\boldsymbol{x}(t) \doteq \hat{\boldsymbol{\theta}}_{\text {future }}^{\text {dev }}(t)\right)$.

In Actor-Critic method, Actor learns optimal control law $\boldsymbol{u}^{\text {actor }}(t)=\mu(\boldsymbol{x}(t))$. Meanwhile, Critic estimates value function $V(\boldsymbol{x}(t))$. We adopt the NGnet (normalized Gaussian network) to implement both Actor and Critic. The estimate of value function is made using the form

$$
V(\boldsymbol{x}(t))=\sum_{k=1}^{N} w_{k}^{C} b_{k}(\boldsymbol{x}(t))
$$

and the $i$ th element of $\boldsymbol{u}^{\text {actor }}(t)$ is determined as

$$
u_{i}^{\text {actor }}(t)=g\left(\sum_{k=1}^{N} w_{i, k}^{A} b_{k}(\boldsymbol{x}(t))+\sigma(t) n_{i}(t)\right)-0.5
$$

where $b_{k}(\boldsymbol{x}(t))$ is $k$ th basis function, $N$ is the number of basis functions, and $w_{k}^{C}$ and $w_{i, k}^{A}$ are the weight for Critic and Actor network, respectively. The function $g(y)$ is the sigmoid function of $y$. Also $n_{i}(t)$ is the white noise for motor command exploration, and $\sigma(t)$ determines the magnitude of noise given by,

$$
\sigma(t)=\sigma_{0} \exp \left(-\frac{V(\boldsymbol{x}(t))}{s_{n}}\right)
$$

where $\sigma_{0}$ and $s_{n}$ are some constant parameters.

To update weights of each network, we adopted the continuous-time version of TD error [14]

$$
\delta(t)=r(t)-\frac{1}{\tau} V(\boldsymbol{x}(t))+\dot{V}(\boldsymbol{x}(t)) .
$$

The weights in Critic are updated by

$$
\dot{w}_{k}^{C}=\beta^{C} \delta(t) e_{k}^{C}(t)
$$

where $\beta^{C}$ is the learning rate and $e_{k}^{C}(t)$ is the eligibility trace

$$
e_{k}^{C}(t)=\int_{0}^{t} \exp \left(-\frac{t-s}{\kappa}\right) b_{k}(s) d s
$$

At the same time, the weights in Actor are updated by

$$
\dot{w}_{i, k}^{A}=\beta^{A} \delta(t) e_{i, k}^{A}(t)
$$

where $\beta^{A}$ is the learning rate and $e_{i, k}^{A}(t)$ is the eligibility trace

$$
\begin{aligned}
e_{i, k}^{A}(t) & =\int_{0}^{t} h(t-s) d_{i, k}(s) d s \\
d_{i, k}^{A}(s) & =\sigma(s) n_{i}(s) b_{k}(\boldsymbol{x}(t))
\end{aligned}
$$

Here function $h(z)$ is the impulse response of the second order low pass filter applied to the motor commands $\boldsymbol{u}$ (see Method for detail).

\section{B. ISM}

The input to ISM is target state $\boldsymbol{\theta}^{\operatorname{trg}}$ and the output of ISM is a set of motor command $\boldsymbol{u}^{i s m}$. We adopted NGnet to implement ISM. The $i$ th element of $\boldsymbol{u}^{i s m}$ is determined by $\boldsymbol{\theta}^{\operatorname{trg}}$ through,

$$
u_{i}^{i s m}=g\left(\sum_{k=1}^{M} v_{i, k} c_{k}\left(\boldsymbol{\theta}^{t r g}\right)\right)
$$

where $c_{k}\left(\boldsymbol{\theta}^{\operatorname{trg}}\right), M$ and $v_{i, k}$ are the $k$ th basis function, the number of basis function and weight respectively.

We applied the Feedback-Error-Learning scheme to the learning process of ISM [17]. Then, feedback controller's output $\boldsymbol{u}^{\text {actor }}(t)$ is used as error signal to ISM network. The updating rule is given by,

$$
\begin{aligned}
\dot{v}_{i, k}(t) & =\eta \tilde{u}_{i}^{\text {actor }}(t) c_{k}\left(\boldsymbol{\theta}^{\text {trg }}\right) \\
\tilde{u}_{i}^{\text {actor }}(t) & =\int_{0}^{t} h(t-s) u_{i}^{\text {actor }}(s) d s
\end{aligned}
$$

where $\tilde{u}_{i}^{a c t o r}(t)$ is $i$ th component of lowpass filtered output of Actor.

\section{FDM}

We adopted a three-layer neural network for implementing FDM. Input signals to the network are current state $\boldsymbol{\theta}(t)$ and lowpass filtered motor commands. The network predicts state change $\Delta \hat{\boldsymbol{\theta}}(t)$ given by

$$
\hat{\boldsymbol{\theta}}_{\text {next }}(t)=\boldsymbol{\theta}(t)+\Delta \hat{\boldsymbol{\theta}}(t) .
$$

Then $n \Delta t$ future state $\hat{\boldsymbol{\theta}}_{\text {future }}(t)$ is predicted by iterating above procedure $n$ time. Note that the value of lowpass filtered motor command is kept constant during the iteration. Teaching signal for the learning process of FDM is actual state $\boldsymbol{\theta}(t+\Delta t)$ observed at next moment. The weights in FDM's network are updated using the back-propagation method.

\section{METHOD}

We applied our motor control-learning model to the simulation of learning process in point-to-point arm reaching task in sagittal plane. After the learning simulation, we reproduced reaching movements of human subject using the trained ISM, FBC and FBC.

\section{A. Learning Simulation}

1) Arm Model: We adopted an arm model consisting of two-link arm with two joints and six muscles (Figure 2). Two joints are shoulder and elbow joints. Six muscles are two pairs of mono-articular muscles and one pair of bi-artricular muscles. In addition to applying low pass filter to motor command, we introduced biological noise in motor command that increases with the mean level of motor command. This noise is called signal-dependent noise [5], and often defined as white noise with zero mean and variance $k u^{2}$.

The motor command including the noise is calculated as,

$$
u_{i}^{\text {noise }}(t)=u_{i}(t)+w_{i} \quad(i=1,2, \cdots, 6)
$$




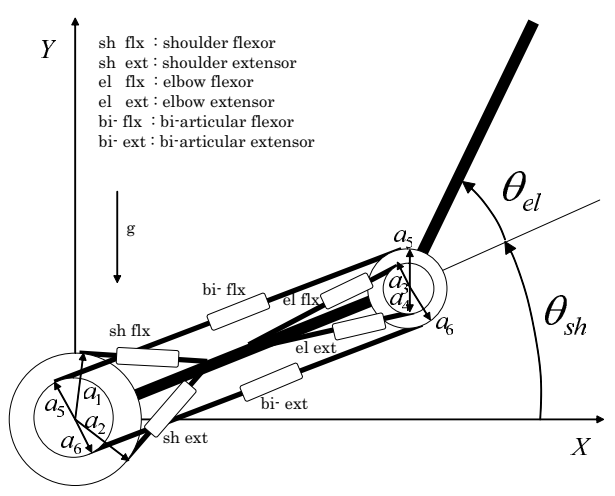

Fig. 2. Two link arm model with two joints and six muscles: $\boldsymbol{\theta}_{s h}$ and $\boldsymbol{\theta}_{e l}$ are the joint angles of shoulder and elbow joints, respectively. $a_{i}(i=$ $1, \ldots, 6)$ is the moment arm of the muscle torque.

where $w_{i} \sim N\left(0,0.01 u_{i}^{2}\right)$.

The motor command $\boldsymbol{u}^{\text {noise }}$ are then filtered by second order lowpass filter [26],

$$
\tilde{u}_{i}=\int_{0}^{t} u_{i}^{\text {noise }}(s) h(t-s) d s \quad(i=1,2, \cdots, 6)
$$

where function $h$ is an impulse response of the filter given by

$$
\begin{aligned}
h(z) & =\frac{1}{\tau_{1}-\tau_{2}}\left(\exp \left(-\frac{z}{\tau_{1}}\right)-\exp \left(-\frac{z}{\tau_{2}}\right)\right) \\
\tau_{1} & =9.26 \times 10^{-2}, \tau_{2}=6.05 \times 10^{-2} .
\end{aligned}
$$

The muscle force $\boldsymbol{T}$ is modeled as

$$
\begin{aligned}
T_{i} & =k_{i}\left(\tilde{u}_{i}\right)\left\{l_{i}^{r e s t}\left(\tilde{u}_{i}\right)-l_{i}\right\}-v\left(\tilde{u}_{i}\right) \dot{l}_{i} \\
k_{i}\left(\tilde{u}_{i}\right) & =k_{0 i}+k_{1 i} \tilde{u}_{i} \\
v_{i}\left(\tilde{u}_{i}\right) & =v_{0 i}+v_{1 i} \tilde{u}_{i} \\
l_{i}^{r e s t}\left(\tilde{u}_{i}\right) & =l_{0 i}+l_{1 i} \tilde{u} \quad(i=1,2, \cdots, 6)
\end{aligned}
$$

where $l_{i}$ is the muscle length and $i_{i}$ is the contraction velocity [28]. Meanwhile, $k_{i}, v_{i}$ and $l_{i}^{\text {rest }}$ denote the muscle stiffness, the muscle viscosity and the rest length of the muscle respectively.

The joint torque $\tau$ is given by,

$$
\boldsymbol{\tau}=A^{T} \boldsymbol{T}
$$

where $A$ is the moment arm matrix. We assumed $A$ is constant.

$$
A=\left(\begin{array}{cccccc}
a_{1} & -a_{2} & 0 & 0 & a_{5} & -a_{6} \\
0 & 0 & a_{3} & -a_{4} & a_{5} & -a_{6}
\end{array}\right)^{T}
$$

2) State Space: In the reaching task, the state space $\boldsymbol{\theta}$ is composed of joint angles and angular velocities of shoulder and elbow joint $\left(\boldsymbol{\theta}=\left(\theta_{s h}, \theta_{e l}, \dot{\theta}_{s h}, \dot{\theta}_{e l}\right)^{T}\right)$.

3) Motor Command: Control signal $\boldsymbol{u}$ is a set of motor commands given to six muscles whose values are set between 0 and 1 . We set the output of ISM between 0 and 1 , and that of Actor between -0.5 and 0.5 . The value of motor command, that is, the sum of $u_{i}^{i s m}$ and $u_{i}^{a c t o r}$ is given by

$$
u_{i}(t)=\left\{\begin{array}{l}
0 \text { for } u_{i}^{i s m}+u_{i}^{\text {actor }}(t)<0 \\
1 \text { for } u_{i}^{\text {ism }}+u_{i}^{\text {actor }}(t)>1 \\
u_{i}^{\text {ism }}+u_{i}^{\text {actor }}(t) \text { otherwise }
\end{array}\right.
$$

4) Reward: The reward value $r(t)$ is determined using two components, $r_{d}$ and $r_{u}$.

The first component $r_{d}$ is the reward for the distance between target position and future hand position obtained from predicted future state $\hat{\boldsymbol{\theta}}_{\text {future }}$. Let us name this distance as "hand distance", and $d(t)$ denotes the distance predicted at time $t$. We determine $r_{d}$ as follows,

$$
r_{d}(d(t))=2\left(\exp \left(-\frac{d^{2}(t)}{\sigma_{d}^{2}}\right)-0.5\right)
$$

where $\sigma_{d}$ is a constant parameter.

The second component $r_{u}$ is the penalty for energy consumption and given by

$$
r_{u}(\tilde{\boldsymbol{u}}(t))=k_{u} \sum_{i=1}^{6} \tilde{u}_{i}^{2}(t)
$$

where $k_{u}$ is some constant.

The total reward is then represented as

$$
r(t)=r_{d}(d(t))-r_{u}(\tilde{\boldsymbol{u}}(t)) .
$$

5) Conditions and Parameter: We set total time of one trial to 2.0 second and terminate each trial if either 2.0 second has past or the value of $\boldsymbol{\theta}$ gets out a certain region. Whole learning simulation consists of 100000 trials.

The initial and target joint angles are chosen randomly within the range $-100^{\circ} \leq \theta_{s h}(0), \theta_{s h}^{t r g} \leq-20^{\circ}, 30^{\circ} \leq$ $\theta_{e l}(0), \theta_{e l}^{\operatorname{trg}} \leq 110^{\circ}$. The initial angular velocities are chosen as $\dot{\theta}_{s h}^{t r g}=\dot{\theta}_{e l}^{t r g}=\dot{\theta}_{s h}(0)=\dot{\theta}_{e l}(0)=0$.

The time step is chosen as $\Delta t=0.01$ second. The renewals of motor command, future state prediction and dynamics are all made for every $\Delta t$. The updating of networks is made for every $\Delta t$. We set $n=15$ and used FDM to predict the state of 0.15 second future.

We set the parameters of reward to $\sigma_{d}=0.06[\mathrm{~m}]$ and $k_{u}=0.1$.

\section{B. Reaching Experiment}

We measured hand trajectories of human reaching movement to compare them with trajectories predicted by our model.

1) Experimental procedure: A right-handed male subject (age 24) generated unconstrained point-to-point reaching movements in sagittal plane. Hand trajectories were measured by 3-dimensional position measurement equipment (OPTOTRAK 3020, NDI). We set seven pairs of initial and target points (figure 3 ), and the subject made 20 movements for each pairs (10 from one point and 10 from the other point). Thus the total number of trials were $140(7 \times 2 \times$ 10). To specify the target points, we marked points on a clear glass located at subject's underarm. Note that unlike 


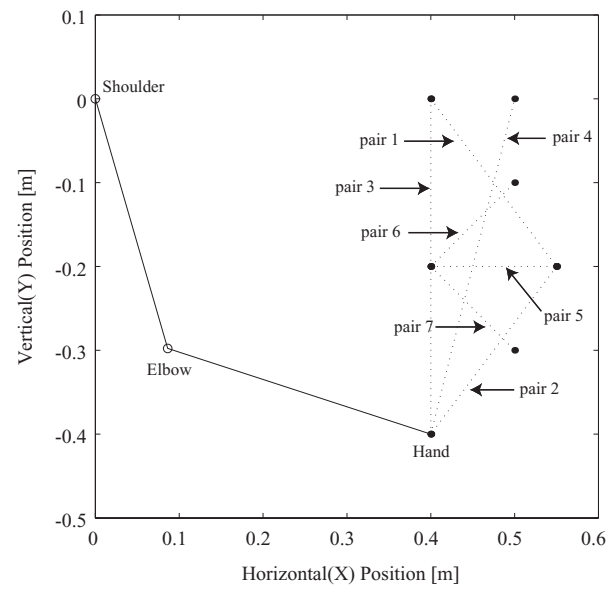

Fig. 3. Seven pairs of initial and target points

figure 3, no straight line was drawn between initial and target points on the glass. No instruction was given about the form of hand path or trajectory execution. Subject made a fist and an optical marker was attached to the back of his hand. We marked a dot at opposite side of the optical marker on subject's hand. We asked subject to make reaching movements so as to overlap the dot marked on his hand to the target points as accurate as possible with natural speed and without rotating his wrist.

2) Data processing: Positional data was digitally lowpass filtered at $10 \mathrm{~Hz}$ using a forth-order Butterworth filter. To generalize the movement duration, we determined the timing of movement onset and offset by a certain threshold of hand velocity. We then interpolated the positional data by the spline curve, and resampled 100 positional data at equally spaced point between movement onset and offset. The resampled trajectory data was averaged over each 10 movement set. We excluded trials whose trajectory deviated widely from the average trajectory. The average of duration between movement onset and offset over movement set was used to calculate the tangential velocity of hand path average.

\section{RESUlts}

\section{A. Learning Simulation Result}

Figure 4(a) shows total reward gained in each trial and average hand distance on each trial. As the number of trial increases, the total reward approaches a certain high value and average hand distance comes close to zero. Therefore, the point-to-point reaching task was successfully accomplished by our model.

Let us see how the three units, ISM, Actor and FDM improved as the learning process proceeds. The results are shown in figure 4(b). The top graph shows the output of ISM in each trial. The middle graph shows Actor's output when $\hat{\boldsymbol{\theta}}_{\text {future }}^{\text {dev }}=\mathbf{0}$ is inputted to Actor. Finally, the bottom graph shows the average errors in FDM's prediction on each trial. Note that $\hat{\boldsymbol{\theta}}_{\text {future }}^{\text {dev }}$ hardly becomes exactly $\mathbf{0}$ during each trial (especially at the early stage of learning process). Thus we (a)
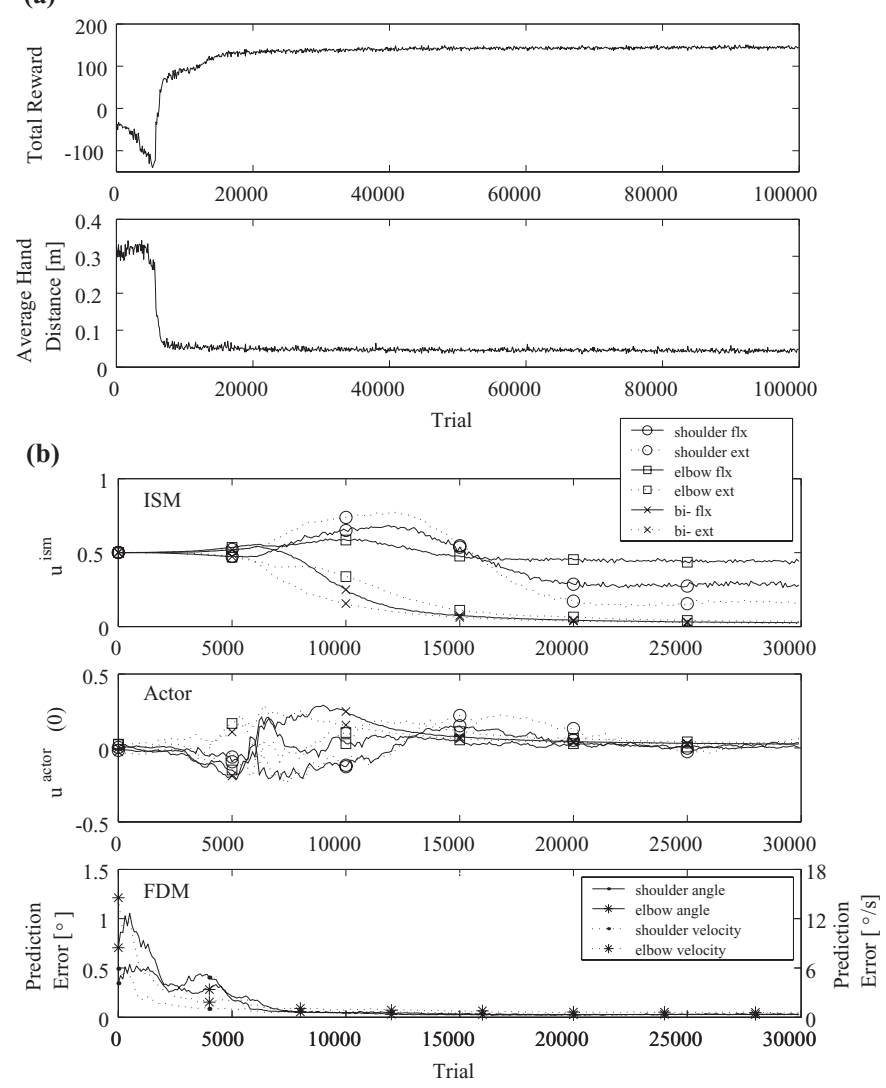

Fig. 4. Learning simulation result. (a) Upper: Total reward gained in each trial, Lower: Average hand distance on each trial. (b) Top: ISM's output in each trial, Middle: Actor's output at the target state, Bottom: FDM's prediction error. The data plotted in (b) are the data from $1^{\text {st }}$ to $30000^{\text {th }}$ trial (notable changes were not found after $30000^{t h}$ trial). Note that each plots are the averages over 100 trials. Actor's output were obtained by inputting $\hat{\boldsymbol{\theta}}_{\text {future }}^{\text {dev }}=\mathbf{0}$ to Actor at the interval between one trial and the next.

get the data of the middle graph by inputting $\hat{\boldsymbol{\theta}}_{\text {future }}^{\text {dev }}=\mathbf{0}$ to the Actor at the interval between one trial and the next. As we can see from figure4(b), the improvements of the three networks were started in order of FDM, Actor and ISM. First of all, FDM became able to predict next state relatively well around $3000^{t h}$ trial. Then Actor, whose input is affected by FDM's prediction, became able to improve its input-output relationship, that is, it started to generate the biased motor commands around $6000^{\text {th }}$ trial. At this stage, arm's movements are driven mainly by Actor's output and the performance is not very good. Finally, the biased motor command of Actor began to improve ISM network.

Although the improvements of FDM, Actor and ISM are started at different timings, the improvement of one unit generates that of other unit. Moreover, the latter improvement promotes the former one. In parallel with the improvement of ISM, Actor began around $25000^{t h}$ trial to stop generating biased motor command at the target point. Furthermore, the prediction error of FDM becomes smaller since the reaching movements get stable and smooth in parallel with the improvements of Actor and ISM network. 


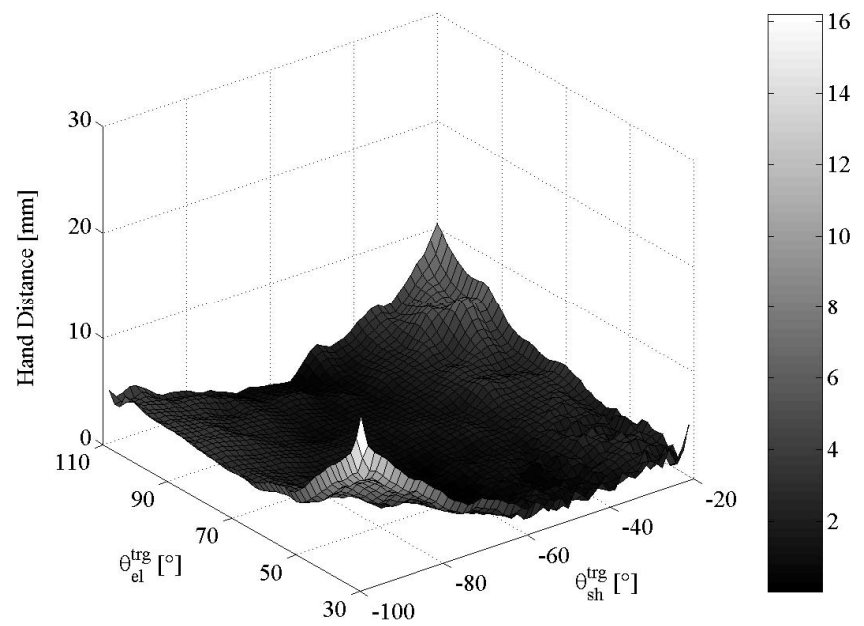

Fig. 5. Hand distance between the target point and the final hand position in 2500 reaching movement trials. $\theta_{s h}^{t r g}$ and $\theta_{e l}^{t r g}$ are the shoulder and elbow joint angles of the target state, respectively. 2500 target states were the points of $50 \times 50$ equally divided grid within the target state range. The values of hand distance are the the distances between target and hand position at the end of reaching movements. Initial state for each reaching movements were chosen randomly within the same range as that of learning simulation. The average and standard deviation are $2.57[\mathrm{~mm}]$ and $1.78[\mathrm{~mm}]$, respectively

\section{B. Hand Distance Accuracy}

To see how accurate hand reaches the target points, we simulated reaching movements toward various points $(2500$ different targets) after 100000 trials for the learning simulation, and measured the distances between the target points and the hand positions when the arm rested. The results are shown in figure 5. For almost all of the targets, the hand reached the points within $10[\mathrm{~mm}]$ around the target. The average distance and the standard deviation are $2.57[\mathrm{~mm}]$ and $1.78[\mathrm{~mm}]$, respectively. Therefore, it can be said that highly accurate reaching movements are achieved based on the successful learning process in our model. Note that the signal-dependent noise was not included to get result shown in figure 5. In the case that the signal dependent noise is included, the average and the standard deviation become $3.37[\mathrm{~mm}]$ and $2.13[\mathrm{~mm}]$. Although the accuracy becomes slightly worse compared to the case that the noise is not included, we can still say it is highly accurate and the learning process has been successfully made in our model under the noisy condition.

\section{Motor Command During Reaching Movement}

To see what kind of muscle activation patterns can be predicted by our model, we show sequences of motor commands during the reaching movements in figure 6 . We simulated upward and downward reaching movements after 100000 trials of learning process. During the upward movement, the flexor muscles first increase their activity, and then activation of the extensor muscles follow them. On the contrary, during the downward movement, the increase in the activation of the extensor muscles is followed by that of flexor muscles. The tonic activation levels are higher in flexor muscles than in extensor muscles, because flexor muscles

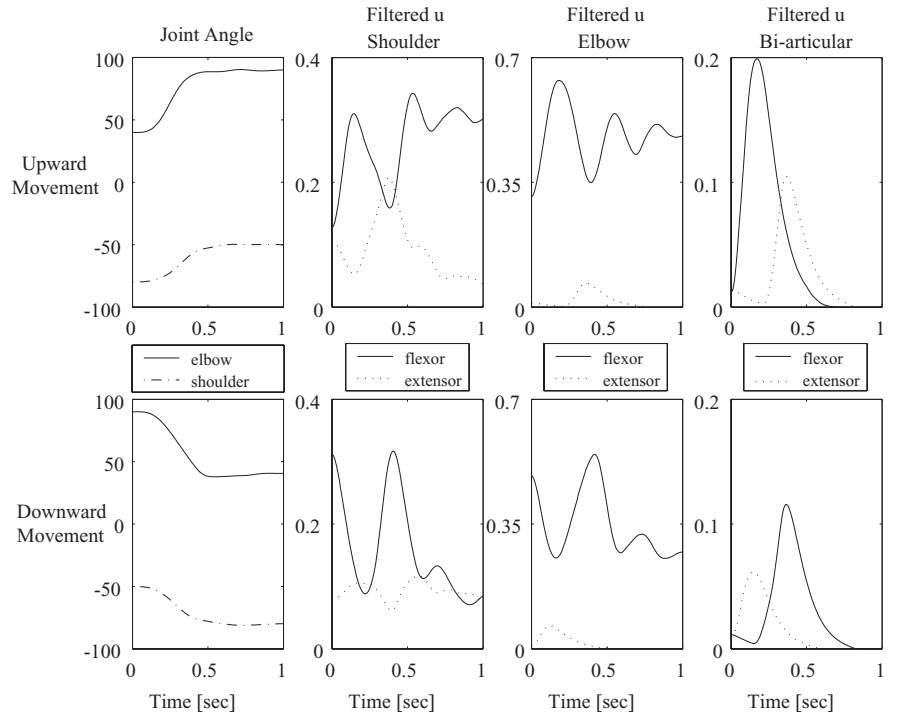

Fig. 6. Motor commands during two reaching movements. Initial and target state for upward movement are $\boldsymbol{\theta}(0)=(-80,40,0,0)$ and $\boldsymbol{\theta}^{\text {trg }}=$ $(-50,90,0,0)$, respectively. Initial and target state for downward movements are the reverse of upward movements. All motor commands plotted are lowpass filtered motor commands.

must generate force against gravity. However, these phasic activation patterns depending on the movement direction are often observed in electromyographic(EMG) pattern in human reaching movements [29][30][31].

\section{Hand trajectory in experiment and simulation}

Point-to-point reaching movements of human subject are simulated by our model using the networks obtained after 100000 trials. The results are shown in figure 7. Initial and target states for the simulation are determined by the average hand positions of subject at the movement onset and offset respectively. Figure 7(a) shows hand paths in the experiment and simulation. The hand paths reproduced by our model are qualitatively in good agreement with those in experiment.

Our model can also reasonably predict the hand speed profiles of subject. As seen in figure 7(b), the smooth and bell-shaped profiles of hand speed are reproduced well. Note that the information given to our model to simulate subject's movement is only about initial and target states. Unlike most of the previous models [3][4][5][8], our model does not need the information about movement duration to generate hand movement. That is, in our model, the movement duration is the result of the movement and it is not determined before the movement is generated. Although our model can not tell exact movement duration before it generates the movements, the movement duration obtained in the simulation resulted in almost the same as the experimental duration. We have not analyzed in detail what made the movement duration in simulation to be as same as the experiment. It is our future work to analyze what kind of parameter makes the movement faster or slower. 

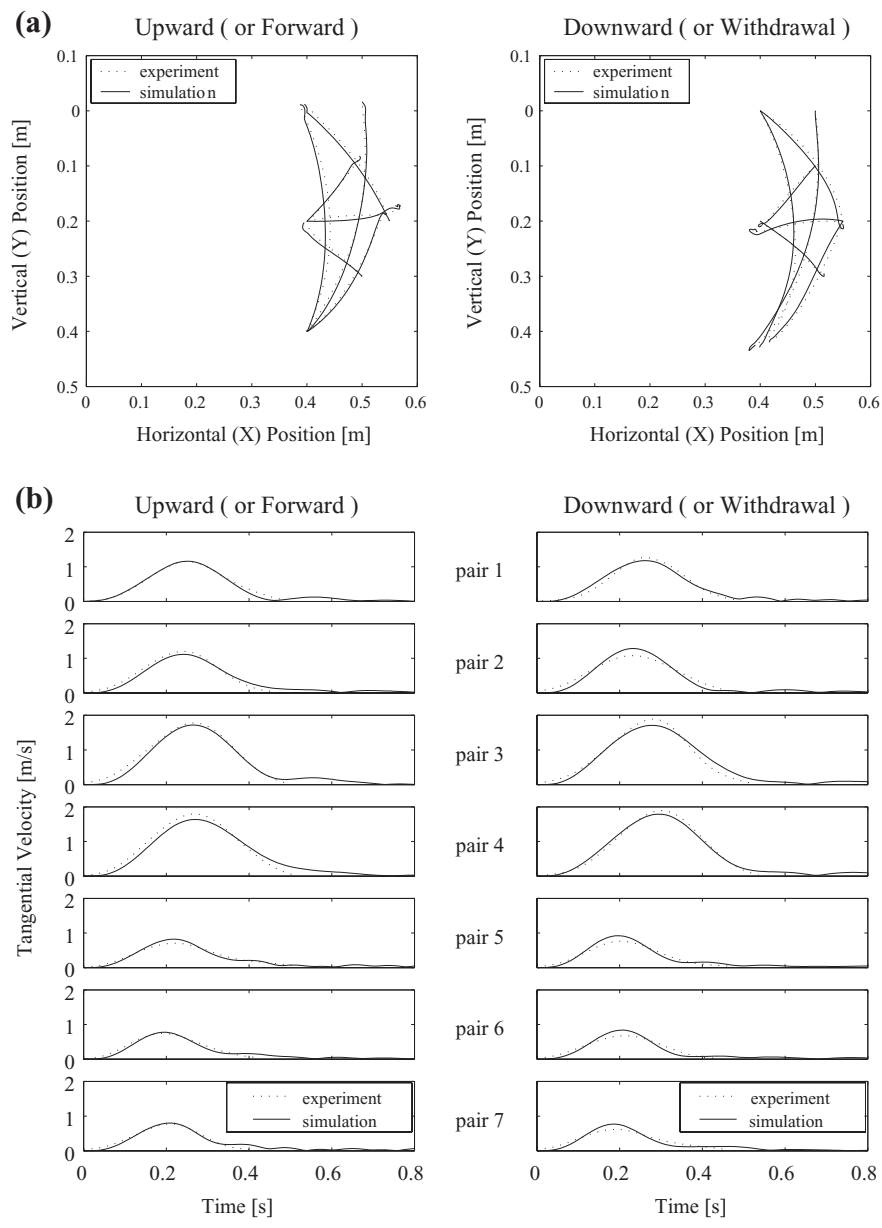

Fig. 7. Hand trajectories in experiment and simulation for seven pairs of initial and target points. (a) Hand paths of human subject and simulation in the upward and downward movements for pair 1,2,3,4,6 and 7, and the forward and withdrawal movements for pair 5. (b) Hand tangential speed profiles of human and simulation trajectories. Hand speed profiles in experiment and simulation are aligned so that the timings of peak velocity become the same.

\section{Conclusions}

In this paper, we proposed a new motor control-learning model that could learn a control law for generating point-topoint reaching movements toward various points in sagittal plane. Unlike previous models, our model could reconstruct reaching movements without prior knowledge of arm dynamics. The only error signal provided to the model was a scalar reward indicating overall task performance. Our simulations demonstrated the ability of the model to learn highly accurate reaching movements in a trial-and-error manner.

The other important feature of our motor control-learning model is that it does not require desired trajectories to generate reaching movements. In our model, the Actor (which is the control unit in the Actor-Critic method) is trained to be a goal-directed feedback controller. On the other hand, the inverse statics model (ISM) serves as a goal-directed feed-forward controller by outputting constant motor commands which set the arm's equilibrium point to coincide with the target. Combining these two goal-directed controllers with a forward dynamics model (FDM) needed to compensate delays, our model was able to predict the hand trajectories of human subjects quite well without planning desired trajectories.

Let us emphasize that the smooth bell-shaped speed profiles do not derive from the smoothness in the parameterization of the Actor and ISM networks or the effect of signal-dependent noise. If we do not assume muscle lowpass filtering, speed profiles show very steep initial rises at movement onset [5][19]. Introducing a second-order filter in the muscle model, however, causes a delay between motor commands and movement. We compensated this delay by using FDM to predict future state instead of current state, and thus avoided increasing the dimensionality of state space. We have not analyzed in detail the effects of using FDM, and in particular how far in the future it should attempt to predict. However, we believe there is a possibility that the CNS is predicting the future state and utilizing it intelligently so as to control our complex body.

There is still an argument on whether the CNS is optimizing desired trajectories and controlling the body to follow them [24], or is optimizing goal-directed feedback controllers and controlling the body directly towards the task goal [8]. Our model can be categorized as the latter concept, and gives a possible solution to the problem of learning the optimal feedback controller. Although we believe that desired trajectories are not planned for our daily movements in general, we are not sure whether such plans exist for welltrained movements such as professional athletes' movements. However, even if we assume that the desired trajectory is optimized for well-trained movements, we still think there must be a prior period that the CNS is learning some controller that can stably follow the desired trajectory. One might imagine that the inverse dynamics model (IDM), acquired by Feedback-Error-Learning, could be used for control. However, as we mentioned in the introduction, the trajectory formation problem is unsolved for Feedback-ErrorLearning. Thus, there is still no complementary model that could explain how the CNS learns optimal desired trajectories for well-trained movements. In the future, we want to extend our model so that it could explain the process of optimal trajectory learning for well-trained movements, and utilize the model to explore whether the CNS is planning the desired trajectory or not.

\section{ACKNOWLEDGMENT}

We thank Professor Emanuel Todorov at University of California San Diego for his helpful discussions and comments. A part of this research was supported by Japan Science and Technology Agency CREST.

\section{REFERENCES}

[1] W. Abend, E. Bizzi and P. Morasso, "Human arm trajectory formation," Brain, vol. 105, pp. 331-348, 1982.

[2] C. G. Atkeson and M. Hollerbach, "Kinematic features of unrestrained vertical arm movements," J. Neurosci., vol. 5, pp. 2318-2330, 1985.

[3] Y. Uno, M. Kawato and R. Suzuki, "Formation and control of optimal trajectory in human multijoint arm movement," Biol. Cybern., vol. 61, pp. 89-101, 1989. 
[4] T. Flash and N. Hogan, "The coordination of arm movements: An experimentally confirmed mathmatical model," J. Neurosci., vol. 5, pp. 1688-1703, 1985.

[5] C. M. Harris and D. M. Wolpert, "Signal-dependent noise determines motor planning," Nature, vol. 394, pp. 780-784, 1998.

[6] T. Flash, "The control of hand equilibrium trajectories in multi-joint arm movements," Biol. Cybern., vol. 57, pp. 257-274, 1987.

[7] H. Miyamoto, E. Nakano, D. M. Wolpert and M. Kawato, "TOPS (task optimization in the presence of signal-dependent noise) model," IEICE Trans. Inform. Syst., vol. J85-DII, pp. 940-949, 2002 (written in Japanese).

[8] E. Todorov and M. Jordan, "Optimal feedback control as a theory or motor coordination," Nature Neurosciene, vol. 5, pp. 1226-1235, 2002.

[9] J. Konczak and J. Dichgans, "The development toward stereotypic arm kinematics during reaching in the first 3 years of life," Exp. Brain Res., vol. 117, pp. 346-354, 1997.

[10] F. T. J. M. Zaal, K. Daigle, L. Gottlieb and E. Thelen, "An unlearned principle for controlling natural movement," J. Neurophysiol., vol. 82, pp. 255-259, 1999.

[11] R. S. Sutton and A. G. Barto, Reinforcement Learning, Cambridge, MA: MIT Press, 1998.

[12] A. G. Barto, "Adaptive critics and the basal ganglia," in Model of information processing in the basal ganglia (Chapter III, pp. 215-232), Edited by J. C. Houk, J. L. Davis and D. G. Beiser, Cambridge, MA: MIT Press, 1995.

[13] K. Doya, "What are the computations of the cerebellum, the basal ganglia and the cerebral cortex," Neural Networks, vol. 12, pp. 961974, 1999.

[14] K. Doya, "Reinforcement learning in continuous time and space," Neural Computation, vol. 12, pp. 219-245, 2000.

[15] J. Izawa, T. Kondo and K. Ito, "Biological arm motion through reinforcement learning," Biol. Cybern., vol. 91, pp. 10-22, 2004.

[16] A. G. Feldman, "Functional tuning of the nervous system during control of movement or maintenace of a steady posture-3. Mechanographic analysis of the execution by man of the simplest motor tasks," Biophysics, vol. 11, pp. 766-775, 1966.

[17] M. Kawato, K. Furukawa and R. Suzuki, "A hierachical neural-network model for control and learning of voluntary movement," Biol. Cybern., vol. 53, pp. 57-66, 1987.

[18] D. M. Wolpert, R. C. Miall and M. Kawato, "Internal models in the cerebellum,” Trends in Cognitive Sciences, vol. 2, pp. 338-347, 1998.

[19] H. Kambara, J. Kim, M. Sato and Y. Koike, "Learning arm's posture control using reinforcement learning and feedback-error-learning," Proc. 26th Annual International Conference of the IEEE Engineering in Medicine and Biology Society, San Francisco, CA, Sept. 2004, pp. 486489.

[20] Y. Wada and M. Kawato, "A neural network model for arm trajectory formation using forward and inverse dynamics models," Neural Networks, vol. 6, pp. 919-932, 1993.

[21] E. Bizzi, A. Polit and P. Morasso, "Mechanisms underlying achievement of final head position," J. Neurophysiol., vol. 39, pp. 435-444, 1976.

[22] E. Bizzi, N. Accornero, W. Chapple and N. Hogan, "Posture control and trajectory formation during arm movement," J. Neurosci., vol. 4, pp. 2738-2744, 1984.

[23] H. Kambara, J. Kim, M. Sato and Y. Koike, "Learning arm's posture control using reinforcement learning and feedback-error-learning," To appear in IEICE Trans. Inform. Syst., 2006 (written in Japanese).

[24] K. Morishige, H. Miyamoto, R. Osu and M. Kawato, "Positional variance on via-point reaching movement supports sequential trajectory planning and execution model," IEICE Trans. Inform. Syst., vol. J87D-II, pp. 716-725, 2004 (written in Japanese).

[25] A. Mannard and R. B. Stein, "Determination of the frequency response of isometric solues muscle in the cat using random nerve stimulation," J. Physiol., vol. 229, pp. 275-296, 1973.

[26] Y. Koike and M. Kawato, "Estimation of dynamic joint torques and trajectory formation from surface electromyography signals using a neural network model," Biol. Cybern., vol. 73, pp. 291-300, 1995.

[27] R. C. Miall and D. M. Wolpert, "Forward models for physiological motro control," Neural Networks, vol. 9, pp. 1265-1279, 1996.

[28] M. Katayama and M. Kawato, "Virtural trajectory and stiffness ellipse during multijoint arm movement predicted by nerural inverse models," Biol. Cybern., vol. 69, pp. 353-362, 1993.
[29] G. L. Almeida, D. Hong, D. Corcos and G. L. Gottlieb, "Organizing principles for voluntary movement : Extending single-joint rules," $J$. Neurophysiol., vol. 74, pp. 1374-1381, 1995.

[30] P. L. Gribble, and D. J. Ostry, "Independent coactivation of shoulder and elbow muscles," Exp. Brain Res., vol. 123, pp. 355-360, 1998.

[31] R. Osu, N. Kamimura, H. Iwasaki, E. Nakano, C. M. Harris, Y. Wada and M. Kawato, "Optimal impedance control for task achievement in the presence of signal-dependent noise," J. Neurophysiol., vol. 92, pp. 1199-1215, 2004. 